\title{
A Clinical Report on the Relationships Between Metabolism and Obesity, Type 2 Diabetes, Cardiovascular Risk by Using the GH-Method: Math-Physical Medicine
}

Gerald C Hsu

EclaireMD Foundation, USA

Introduction

By using the GH-Method: math-physical medicine and big data on one particular patient (the author), this clinical paper describes the relationship between his metabolic state and medical conditions, including obesity, type 2 diabetes (T2D), and cardiovascular risk.

\section{Method}

The obese patient was diagnosed with T2D, hyperlipidemia, and hypertension over 25 years ago and suffered five cardiac episodes from 1994 to 2006 . For this study, approximately $1.5 \mathrm{M}$ detailed metabolic conditions and lifestyle data $(1 / 1 / 2012$ - 12/31/2018) were collected and processed; advanced mathematics, physics concepts, engineering modeling, and artificial intelligence (AI) were utilized
*Corresponding author

Gerald C Hsu, EclaireMD Foundation, USA

Results:

\begin{tabular}{|c|c|c|c|c|c|c|}
\hline & 2000 & 2010 & 2012 & 2017 & 2018 & Note: \\
\hline \multicolumn{7}{|l|}{ Metabolism } \\
\hline MI \& GHSU & & & $102 \%$ & $57.4 \%$ & $56.6 \%$ & $73.5 \%$ is "break-even" line \\
\hline \multicolumn{7}{|l|}{ Lifestyle Management } \\
\hline Food Quantity (\% of normal portion) & & $112 \%$ & & $85 \%$ & $84 \%$ & \\
\hline Daily Exercise (Walking steps) & & 2,000 & & 17,863 & 18,292 & \\
\hline Post-Meal Walking (steps) & & 500 & & 4,440 & 4,570 & \\
\hline \multicolumn{7}{|l|}{ Obesity } \\
\hline Weight (Ibs) & 220 & 210 & 196 & 174 & 171 & took 3 years efforts \\
\hline Waistline (inches) & 46 & 44 & 44 & 33 & 32 & took 5 years efforts \\
\hline BMI & 32.7 & 31.2 & 29.1 & 25.8 & 25.4 & \\
\hline \multicolumn{7}{|l|}{ Diabetes } \\
\hline Daily Glucose (mg/dL) & & 279 & & 117 & 116 & Using glucose prediction models \\
\hline A1C & & $10.0 \%$ & & $6.5 \%$ & $6.5 \%$ & \\
\hline \multicolumn{7}{|l|}{\begin{tabular}{|l} 
Chronic Diseases \\
\end{tabular}} \\
\hline SBP & & 150 & 127 & 106 & 106 & \\
\hline DBP & & 100 & 85 & 64 & 64 & \\
\hline Triglyceride $(<150)$ & & 1161 & & 115 & 85 & \\
\hline HDL (>40) & & 24 & & 45 & 49 & \\
\hline LDL $(<130)$ & & 174 & & 99 & 109 & \\
\hline ACR (Kidney $<30$ ) & & 116 & & 25 & 15 & High ACR was the "wake-up call" \\
\hline Cadiovascular Risk & & & & & & 5 heart episodes (1994 - 2006) \\
\hline Heart Attack or Stroke (\%) & $74 \%$ & & $62 \%$ & $26.4 \%$ & $31 \%$ & Framingham's 2017 is $26.7 \%$ \\
\hline
\end{tabular}

Table 1: Chronic diseases, including obesity, T2D, hyperlipidemia, hypertension show significant improvements when metabolism improved 


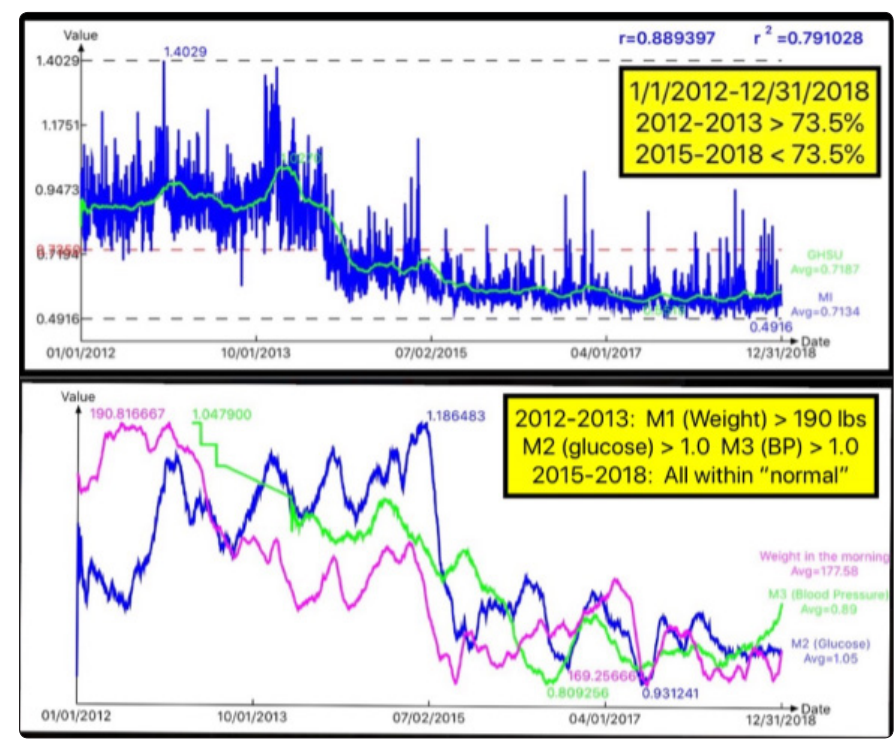

Figure 2: Relationship between Metabolism, including MI, GHSU and chronic diseases

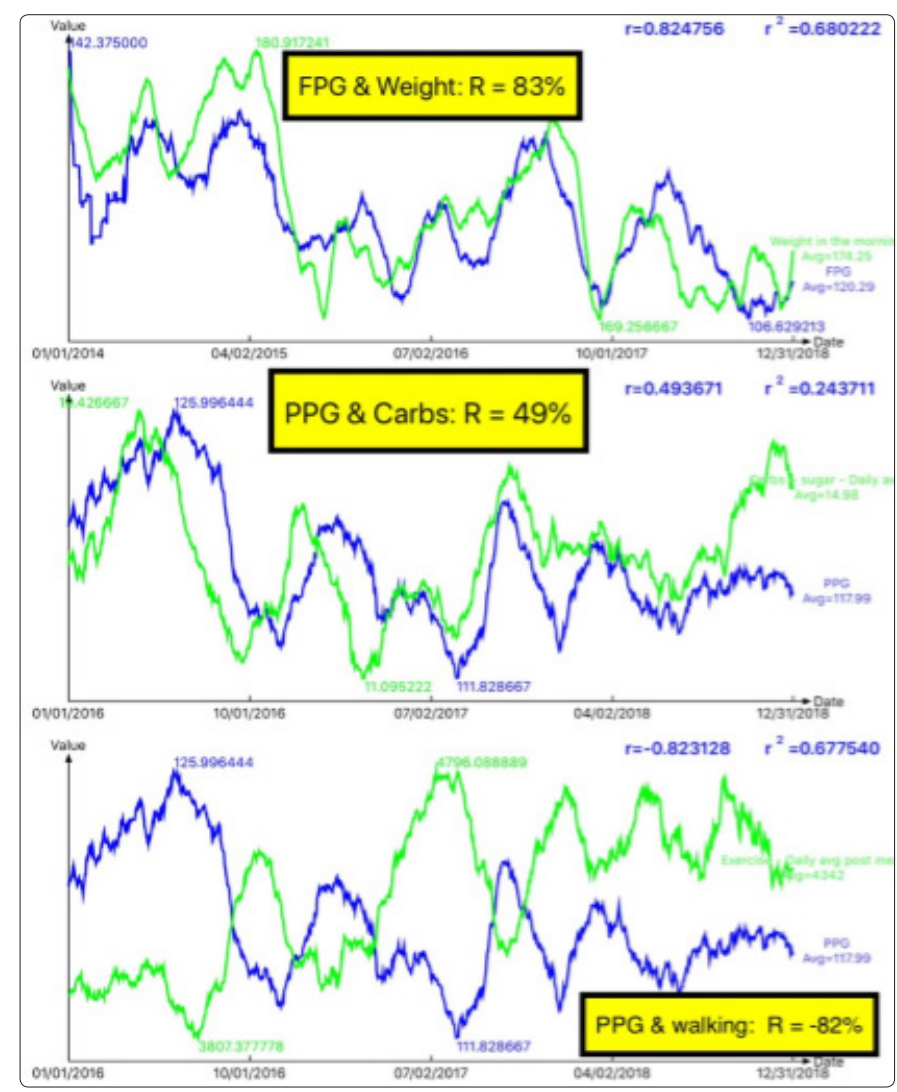

Figure 3: Relationship between T2D and weight, food, and exercise

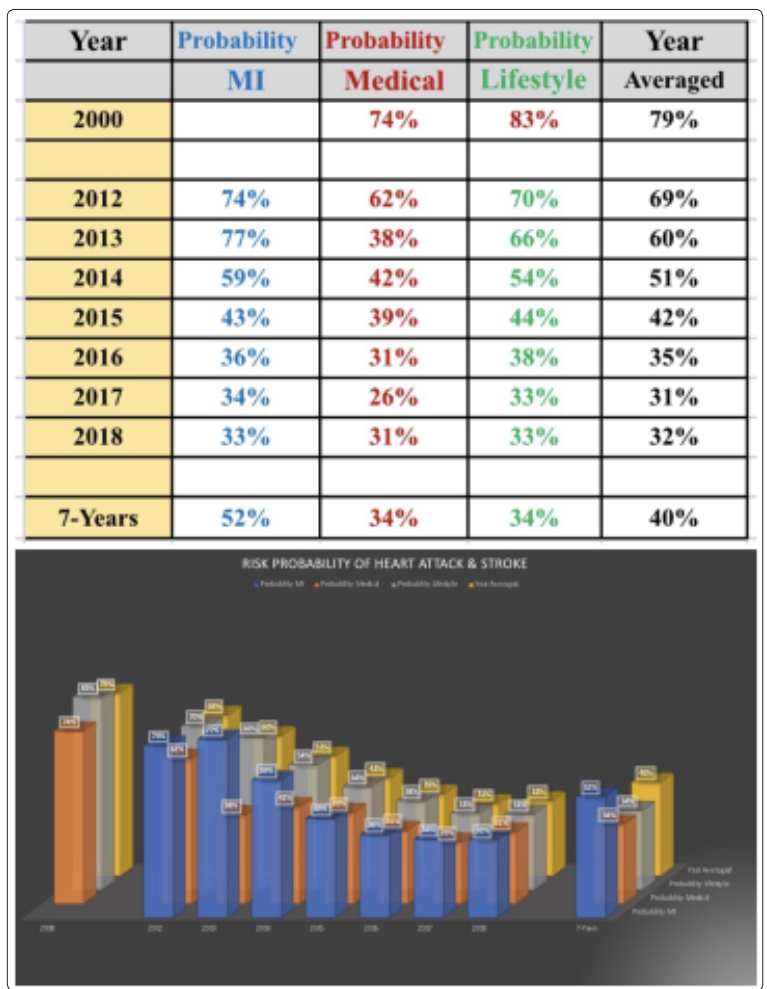

Figure 4: Risk probability of having a heart attack or stroke reducing significantly

\section{Conclusion}

This math-physical medicine approach has quantitatively proven the close relationship between metabolic changes due to lifestyle improvement and effective chronic disease control.

\section{Referesces}

1. Hsu Gerald C (2018) Using Math-Physical Medicine to Control T2D via Metabolism Monitoring and Glucose Predictions. Journal of Endocrinology and Diabetes 1: 1-6.

2. Hsu Gerald C (2018) Using Math-Physical Medicine to Analyze Metabolism and Improve Health Conditions. Video presented at the meeting of the 3rd International Conference on Endocrinology and Metabolic Syndrome.

3. Hsu Gerald C (2018) Using Signal Processing Techniques to Predict PPG for T2D. International Journal of Diabetes \& Metabolic Disorders 3: 1-3.

4. Hsu Gerald C (2018) Using Math-Physical Medicine and Artificial Intelligence Technology to Manage Lifestyle and Control Metabolic Conditions of T2D. International Journal of Diabetes \& Its Complications 2: 1-7.

5. Hsu Gerald C (2018) A Clinic Case of Using Math-Physical Medicine to Study the Probability of Having a Heart Attack or Stroke Based on Combination of Metabolic Conditions, Lifestyle, and Metabolism Index. Journal of Clinical Review \& Case Report 3: 1-2.

Copyright: (02020 Gerald C Hsu, et al. This is an open-access article distributed under the terms of the Creative Commons Attribution License, which permits unrestricted use, distribution, and reproduction in any medium, provided the original author and source are credited. 